Copyright (2011) Society of Photo-Optical Instrumentation Engineers. One print or electronic copy may be made for personal use only. Systematic reproduction and distribution, duplication of any material in this paper for a fee or for commercial purposes, or modification of the content of the paper are prohibited. 


\section{Nanoscale plasmonic resonators with high Purcell factor: spontaneous and stimulated emission}

Ewa M. Goldys, Wei Deng, Nils P. Calander, K. Drozdowicz-Tomsia, Dayong Jin

Ewa M. Goldys, Wei Deng, Nils P. Calander, K. Drozdowicz-Tomsia, Dayong Jin, "Nanoscale plasmonic resonators with high Purcell factor: spontaneous and stimulated emission," Proc. SPIE 7909, Colloidal Quantum Dots/ Nanocrystals for Biomedical Applications VI, 79090H (12 February 2011); doi: 10.1117/12.881170

SPIE. Event: SPIE BiOS, 2011, San Francisco, California, United States 
Invited Paper

\title{
Nanoscale plasmonic resonators with high Purcell factor: Spontaneous and stimulated emission
}

\author{
Ewa M. Goldys, Wei Deng, Nils P. Calander, K. Drozdowicz-Tomsia, and Dayong Jin \\ MQ BioFocus Research Centre, Macquarie University, 2109 North Ryde, NSW, Australia
}

\begin{abstract}
Plasmonic nanoparticles with silver cores and silica shells containing Eu fluorophores near the surface have been produced by wet chemistry method and their spontaneous emission properties characterized. Fluorescence amplification and decreased lifetime is interpreted within the Purcell framework which highlights the role of surface plasmon polariton modes of the nanoparticle. These behave as energy-storing resonators, with values of the $\mathrm{Q}$ factor between 50 and 170 at the fluorophore wavelength of $615 \mathrm{~nm}$, and very small mode volumes, in the order of $10^{4} \mathrm{~nm}^{3}$, producing high Purcell factors of over 4000. Comparison of experiment with theoretical calculations by using the Mie theory shows that the values of cavity Q factors are moderated by the nonradiative rate of fluorophore molecules close to metal. The criteria for laser action in such composite nanoparticles are also presented, including lasing frequencies and threshold gain.
\end{abstract}

Keywords: plasmonic nanoparticles, fluorescence enhancement, Purcell factor, lasing threshold

1. INTRODUCTION

Optical cavities with their ability to strongly modify photon density of states have been attracting attention of the research community for several decades. The emergence of plasmonics has brought to the fore high level of localization of electromagnetic field in metals and significantly reduced wavelengths. This made it possible to explore plasmonic cavities supporting ultrasmall mode volumes, of great interest for applications such as sensing molecular-size substances ${ }^{1}$, coupling to single "atom" emitters for single photon sources ${ }^{2}$, enhanced local nonlinearities ${ }^{3}$ and strong coupling with atomic systems ${ }^{4}$. The extent of the analogy between classical macroscopic cavities and nanoscale cavities in plasmonic systems has only recently been realised ${ }^{5,6}$. Metal and metal-dielectric structures store energy in plasmonic oscillations, and are hence endowed with a $\mathrm{Q}$ factor, albeit small and limited by geometry-independent dielectric functions of the metal and the medium. Phase retardation is also possible in the presence of metal-dielectric interfaces in composite nanostructures, in complete analogy with traditional optical cavities. As a consequence, an increased fraction of electromagnetic energy may be stored in the field increasing the $Q$ factor and strengthening photon density of states within and near the nanostructure. This increased photon density of states has major implications for the behaviour of fluorophores located in the nanostructure. They experience modified radiative and nonradiative rates which can combine to yield increased brightness, improved photostability and reduced fluorescence lifetimes. The extent of these modifications is exacerbated by ultrasmall mode volume, as a result of which composite metal-dielectric nanostructures can show high Purcell factors, comparable to high Q semiconductor micropillar cavities ${ }^{7}$. Furthermore, their Q factor is sufficient for lasing under suitable conditions. Here we report experimental and theoretical investigations of composite metal-dielectric nanostructures that show these extreme properties.

2.

EXPERIMENT

\subsection{Synthesis}

The structures had silver cores and dielectric $\left(\mathrm{SiO}_{2}\right)$ shells coated with an ultrathin second $\mathrm{SiO}_{2}$ shell doped with a lanthanide chelate BHHCT-Eu-DPBT ${ }^{8}$ (see Figure 1 a). First, silver nanoparticle colloids were prepared by reduction of silver nitrate with trisodium citrate ${ }^{9}$. Freshly prepared $10 \mathrm{ml}$ of $1 \%$ trisodium citrate was added dropwise within 2 min into $490 \mathrm{ml}$ of boiling aqueous solution containing $90 \mathrm{mg}$ of $\mathrm{AgNO}_{3}$ under vigorous stirring. After boiling for 1 hour, the reaction solution was cooled to room temperature. The as-prepared silver colloid solution was centrifuged at $500 \mathrm{rpm}$ for 1 hour to remove larger colloids. Silver colloids with different sizes were prepared from above initial colloids by using the following procedure. $20 \mathrm{ml}$ of initial silver colloids was added to $380 \mathrm{ml}$ of boiling Nanopure water followed

*ewa.goldys@mq.edu.au; phone 6129850 8902; fax 6129850 8115; http://www.physics.mq.edu.au/ goldys/core

Colloidal Quantum Dots/Nanocrystals for Biomedical Applications VI, edited by Wolfgang J. Parak, Kenji Yamamoto, Marek Osinski, Proc. of SPIE Vol. 7909, 79090H · (C) 2011 SPIE · CCC code: 1605-7422/11/\$18 · doi: 10.1117/12.881170 
by the addition of $90 \mathrm{mg}$ of $\mathrm{AgNO}_{3}$. Subsequently, $10 \mathrm{ml}$ of $1 \%$ trisodium citrate was added dropwise to the suspension which was kept boiling for $30 \mathrm{~min}$. This procedure leads to the formation of colloids with different silver core sizes. After cooling to room temperature, the final suspension was firstly centrifuged at $500 \mathrm{rpm}$ for 1 hour to separate larger Ag colloids followed by the second-round centrifugation of supernatant at $2000 \mathrm{rpm}$ for 1 hour to obtain the medium size Ag nanoparticles. In order to obtain smaller Ag colloids, the supernatant obtained after second-round centrifugation was then centrifuged at $5000 \mathrm{rpm}$ for $10 \mathrm{~min}$. In this way three Ag suspension with different Ag diameters of $33 \pm 6 \mathrm{~nm}$, $52 \pm 8 \mathrm{~nm}$ and $82 \pm 9 \mathrm{~nm}$ were obtained and used for further experiments.

Further, $\mathrm{Ag} / \mathrm{SiO}_{2}$ nanocomposites were prepared using a method reported by Liu et al ${ }^{10}$, with some modifications. Under vigorous stirring, $50 \mathrm{ml}$ of the silver colloids solution was mixed with $200 \mathrm{ml}$ of ethanol followed by the addition of $4 \mathrm{ml}$ of $30 \%$ ammonium hydroxide. After alkaline initiation, different amounts $(2.5,5$ or $10 \mathrm{ml})$ of $10 \mathrm{mM}$ TEOS ethanol solution were added dropwise to the suspension. The reaction was stirred at room temperature for 24 hours. Each suspension of silica-coated silver colloids was centrifuged and washed 3 times with ethanol, followed by resuspension in $50 \mathrm{ml}$ ethanol. The thickness of the silica shell was determined from TEM images and varied from $\sim 10 \mathrm{~nm}$ to $~ 70 \mathrm{~nm}$. The subsequent covalent conjugation of BHHCT-Eu-DPBT with such $\mathrm{Ag} / \mathrm{SiO}_{2}$ nanocomposites was achieved by using the following method. Typically, $500 \mu \mathrm{L}$ of $2 \mathrm{mg} / \mathrm{ml}$ APS-BHHCT-Eu-DPBT toluene solution was added dropwise to 10 $\mathrm{ml}$ of $\mathrm{Ag} / \mathrm{SiO}_{2}$ ethanol solution under vigorous stirring followed by the addition of $0.24 \mathrm{ml}$ of $30 \%$ ammonium hydroxide. After stirring for 20 minutes, $300 \mu \mathrm{L}$ of $10 \mathrm{mM}$ TEOS ethanol solution was added dropwise into the suspension to form the second silica shell. The reaction was allowed to continue for 24 hour at room temperature (see Figure $1 \mathrm{~b}$ ). The Eu chelate-doped $\mathrm{Ag} / \mathrm{SiO}_{2}$ nanocomposites were centrifuged and washed 3 times with ethanol to remove excessive APS-BHHCT-Eu-DPBT, followed by resuspension in $5 \mathrm{ml}$ ethanol. Measurements were performed on these stock suspensions.

Finally, BHHCT-Eu-DPBT-doped hollow silica nanoshells were prepared from the corresponding dye-doped $\mathrm{Ag} / \mathrm{SiO}_{2}$ nanocomposites by using the following strategy. $2 \mathrm{ml}$ of $0.1 \mathrm{M}$ sodium cyanide solution was added to $1 \mathrm{ml}$ of BHHCTEu-DPBT-doped $\mathrm{Ag} / \mathrm{SiO}_{2}$ nanocomposites with gentle stirring overnight to ensure complete etching of silver cores from those nanocomposites. The resulting hollow silica nanoshells were centrifuged and washed 3 times with ethanol, followed by resuspension in $1 \mathrm{ml}$ ethanol. Since the Eu chelates were covalently doped in the second silica layer, the etching of silver core with cyanide ions did not cause the leakage of the chelates from the silica layer. This procedure permits the same amount of the chelates to be excited with and without silver cores, allowing more precise evaluation of the effect of silver nanoparticles than other methods by using pure silica nanoparticles as reference.

\subsection{Characterization}

Nanoparticle morphology was characterized by transmission electron microscopy (TEM). The TEM images were taken on a PHILIPS CM10 system at an accelerating voltage of $100 \mathrm{kV}$. The samples were prepared by placing a drop of dilute ethanol dispersion of the nanocomposites and nanoshells on the surface of a copper grid. Fluorescence spectra of samples in the solution were obtained using a Fluorolog-Tau-3 system with 450W Xe lamp excitation. The spectral width was set to $8 \mathrm{~nm}$. The emission spectra were excited at $350 \mathrm{~nm}$ and recorded over a range of 560-670 $\mathrm{nm}$. Fluorescence lifetime was measured using a purpose-built UV epi-fluorescence microscopy system $(\times 10$ objective; dichroic beam splitter (Zeiss, FT395)). A high-power UV LED with $\sim 250 \mathrm{~mW}$ power at $365 \mathrm{~nm} \angle \lambda=10 \mathrm{~nm}$ ) was used for excitation. In this work the UV LED was operated in a pulsed mode ( $1 \mathrm{kHz}, 100 \mu \mathrm{s}, 10 \%$ duty cycle). The values of lifetime were derived by a bi-exponential curve fitting (Origin 8.0 software) with the residual error function $\chi^{2}$ value of less than 1.0.

\subsection{Calculations}

\subsubsection{Calculations of Purcell factor}

The calculations were performed at a computer cluster with 15 nodes, with each node based on an Intel Xeon chip E5530 @ 2.4GHz, with 8MB cache and 16 GB of RAM. The Comsol program version 3.5a, with the RF module, in its threedimensional version was used for all simulations. The Comsol program is based on the Finite Element Method (FEM), and the RF module applies it to electromagnetic calculations. The FEM scheme used in this work employs nonorthogonal grids which resolve the problem of "spurious" solutions, frequent in calculations with rectangular grids. The refractive index of the silver used in this work was taken from Ref. 11. The calculations were carried out for the boundary condition of a plane polarized electromagnetic wave at normal incidence arriving from above, and the upper and lower boundaries of the simulated regions were set to absorbing. Absorbing boundaries were also set at the structure sides. The FEM calculations of the electric field in the structures have been validated by the Mie theory. These Mie solutions have been also used to evaluate average electric fields at the fluorophore location. 


\subsubsection{Lasing criteria}

We evaluated the resonance frequencies of the dipolar and higher modes in the nanoparticles by finding nontrivial solution of the Maxwell's equations that is finite at the centre of the core and tends to zero at infinity. The lasing frequency is determined by solving a system of equations derived from matching the tangential components of the electric and magnetic fields at the boundaries of the inner and outer surface of the spherical shell, in a way similar as in References 12 and 13. This matching is possible only for selected values of the wave vector of the spherical wave, which specifies the condition for real and imaginary part of a dielectric function, for each frequency.

In the calculations shown here we used the bulk dielectric function in the metal from the work by Johnson and Christy ${ }^{14}$ which ignores the effect of "mean free path" correction applicable to nanoscale metals. However Nordlander demonstrated that these corrections are not necessary to accurately model gold nanoparticles, and that the Johnson and Christie dielectric function is adequate ${ }^{15}$. Nonlocal effects have also been ignored, as they begin to become pronounced only at a single nanometre scale ${ }^{16}$.

On a more detailed level, the calculation of the eigenmodes of a structure made of a core surrounded by a concentric shell placed in the medium is carried out in similarly as in Reference 17. The electromagnetic field in each of the regions is expressed in terms of spherical harmonics. Since the field has to be finite in all points inside the shell core, we use the spherical Bessel function of the first kind of the $l$-th order, $j_{l}\left(k_{1} r\right)$, where $l$ is the orbital momentum quantum number, in

order to describe the field of field of the $l$-th spherical harmonic inside the shell core. Here $k_{1,2,3}=\omega \sqrt{\frac{E_{1,2,3}}{c}}$, and $c$ is the speed of light. Inside the shell layer we use a combination of the spherical Hankel functions of the first and second kind, $h_{l}(1)\left(k_{2} r\right)$ and $h_{l}(2)\left(k_{2} r\right)$, respectively. The solution in the outside medium is sought as a spherical Hankel function $h_{l}\left(k_{3} r\right)$ because it does not diverge at infinity, while its counterpart does. We impose the usual continuity conditions for the components of the electric and magnetic fields at internal and external surfaces of the shell. Enforcing the condition that we have only an outgoing wave in the medium determines the wave vector $k_{3}$ for each $l$ and hence the frequency of the eigenmode (wavelength) $E_{l m n}$. These are degenerate with respect to the quantum number $m$ due to spherical symmetry, so we can take $m=0$. In most cases we only consider the modes with the radial quantum number $n=1$, and only in some cases $n=2$ because only such modes can be excited in a structure with radius that is smaller than the light wavelength. The dipole (quadrupole, etc) nodes correspond to $l=1$ (2, 3,etc)

Our analytical calculations have been confirmed by numerical solution of the Maxwell's equations by using COMSOL software.

3.

\section{RESULTS AND DISCUSSION}

\subsection{Spontaneous emission}

Typical emission spectra of BHHCT-Eu-DPBT in Ag@SiO ${ }_{2}$ nanocomposites (excited at below $1 \mathrm{~mW} / \mathrm{cm}^{2}$ ) with Ag core size of $25 \mathrm{~nm}$ and silica shell thickness of $25 \mathrm{~nm}$, as well as the control samples without Ag core are presented in Figure $1 \mathrm{~b}$. The observed characteristic luminescence lines of the $\mathrm{Eu}^{3+}$ ion at around 580, 590, 614, 650 and a weak one at 700 $\mathrm{nm}$, correspond to ${ }^{5} D_{0} \rightarrow{ }^{7} F_{J}$ transitions $(J=0,1,2,3$ and 4 , respectively). The spectral shape of the emission in such nanocomposites was unchanged compared to Eu chelate in ethanol solution. The fluorescence enhancement factors (Figure $1 \mathrm{c}$ ) have been obtained by comparing the fluorescence intensities at the most intense $614 \mathrm{~nm}$ peak $\left({ }^{5} D_{0} \rightarrow{ }^{7} F_{2}\right.$ transition) for samples with different Ag-core sizes and $\mathrm{SiO}_{2}$ shell thickness with those corresponding to control samples without the metal core but with the same nanoparticle concentrations. We observed fluorescence enhancement of BHHCT-Eu-DPBT that depends both on the size of Ag core and metal-fluorophore distance. For example, our results show that in the samples fixed core size of $52 \mathrm{~nm}$ but with different $\mathrm{SiO}_{2}$ shell thickness, an over tenfold (10.7) fluorescence enhancement was observed for the silica shell thickness of $12 \mathrm{~nm}$ and 9.5 for the $25 \mathrm{~nm}$ shell This is contrary to commonly accepted models that have been developed for planar geometries ${ }^{18}$, which do not capture the dependence on the size of the Ag core. The fluorescence enhancement factors at shorter wavelengths of $580 \mathrm{~nm}$ and 589 $\mathrm{nm}$ were relatively higher compared to that $614 \mathrm{~nm}$, with a marked decrease observed at $652 \mathrm{~nm}$ (Figure $1 \mathrm{~d}$ ). This is consistent with the fact that a relatively stronger MEF effect is observed for wavelengths closer to the plasmon resonance for the examined nanoparticles, which are in the range of 350-470 nm, depending on geometry. 
The measurements of fluorescence lifetime have also been carried out, confirming the metal-enhanced fluorescence effect, which is characterized by increase of fluorescence intensity accompanied by a decrease in the lifetime of a fluorophore located in the proximity of the metallic nanostructures ${ }^{19-21}$. To this aim fluorescence decay curves were acquired for samples with and without $\mathrm{Ag}$ cores, and results show that $\mathrm{Ag} @ \mathrm{SiO}_{2}$ nanocomposites exhibit shorter lifetimes compared to the corresponding hollow silica nanoshells of 212 us (Table 1). The highest decrease of the BHHCT-Eu-DPBT fluorescence decay time (from $301 \mu$ s for ethanol solution of BHCCT to $60 \mu \mathrm{s}$ in the composite nanoparticle) was measured for the shortest metal-fluorophore distance (shell thickness) of $12 \mathrm{~nm}$ and the Ag core size of $52 \mathrm{~nm}$.

a)

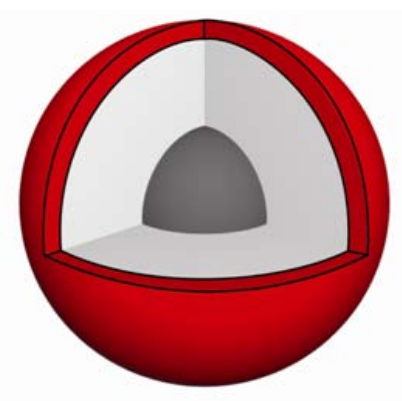

c)

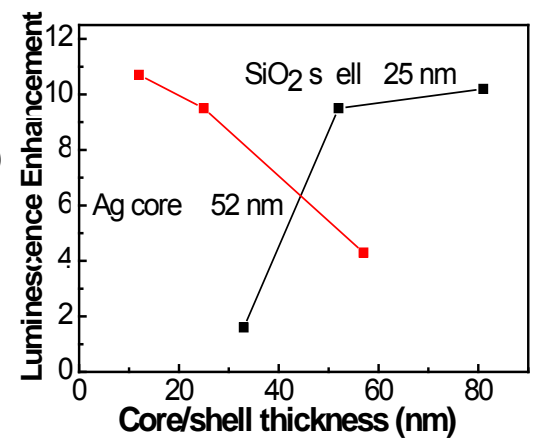

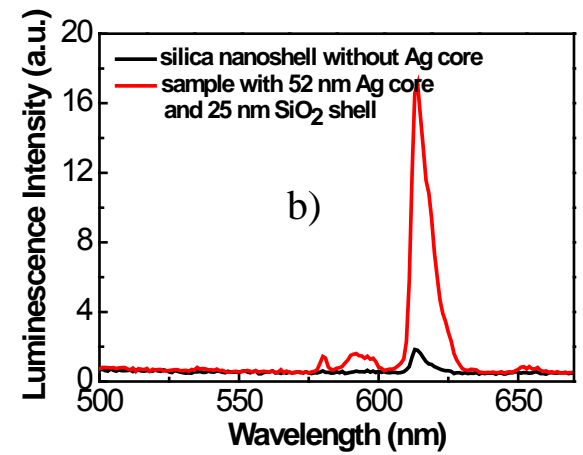

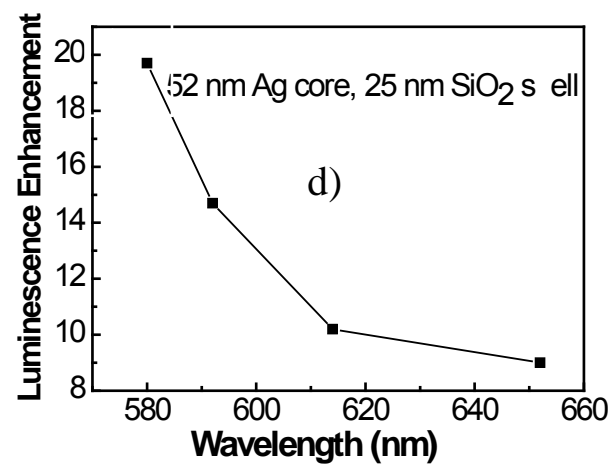

Figure 1. a) Schematics of $\mathrm{Ag} / \mathrm{SiO}_{2}$ nanocomposites doped with BHHCT-Eu-DPBT. Dark grey -Ag core, light greyfirst $\mathrm{SiO}_{2}$ shell, red - second $\mathrm{SiO}_{2}$ shell, $<5$ nm thick, doped with BHHCT-Eu-DPBT. b) example fluorescence spectrum of a $52 \mathrm{~nm}$ core $25 \mathrm{~nm}$ shell structure (red) and a corresponding structure without Ag core (black, c) enhancement factor for different geometries measured at $614 \mathrm{~nm}$. Red: Ag core $=52 \mathrm{~nm}$, different shell thicknesses, black: $\mathrm{SiO}_{2}$ shell of $25 \mathrm{~nm}$, different core radii. d) enhancement factor at different wavelengths for a $52 \mathrm{~nm}$ core $25 \mathrm{~nm}$ shell structure.

\subsection{Purcell factor}

The fluorescence intensity and fluorescence lifetime are determined by its spontaneous radiative rate. The spontaneous emission rate ${ }^{\Gamma}$ of a fluorophore located at $r$ emitting at a single frequency $\omega$ is given by the Fermi Golden Rule:

$$
\Gamma(r)=2 \pi / \hbar\langle d * E(r))\rangle^{\top} 2 \rho(\omega)
$$

where $\boldsymbol{d}$ is the electric dipole of the transition, $\boldsymbol{E}(\boldsymbol{r})$ is the local rms electric field and $\rho(\omega)$ is the density of electromagnetic modes at the frequency $\omega$ that are available for this spontaneous decay. The density of electromagnetic modes can be controlled by placing the fluorophore in a suitably tailored dielectric environment, especially when this environment has modes that are resonant with the fluorophore transitions. An example of such environment is a resonant cavity with a (high) quality factor $Q$ which amplifies the spontaneous radiative rate by a Purcell factor $F$ 22. 


$$
F=\frac{3 Q\left(\frac{\lambda_{c}}{n}\right)^{3}}{4 \pi^{2} V_{e f f}}
$$

The Purcell factor is a simple and attractive figure of merit for cavity QED, and it has been widely used with dielectric cavities including microdisks ${ }^{23}$ and photonic crystals ${ }^{24}$, and for predictions of their lasing thresholds. With the realization of first plasmonic resonators, this concept is currently being expanded ${ }^{25}$ to include plasmonic systems ${ }^{26}$. This is based on the idea that metal -dielectric composite system can function as plasmonic optical cavity which stores energy mainly in the enhanced field intensity of the electromagnetic modes ${ }^{27}$. However, in order for the conventional Purcell formalism to apply to metal nanostructures, it needs to be recast to properly account for ohmic and radiative losses in plasmonic structures. Our analysis is applicable to arbitrary modes of the surface plasmon polaritons, and we ignore retardation effects, as well as small contributions from magnetic field energy.

In cavities with ohmic and radiative losses we need to modify the $Q$ factor of the relevant electromagnetic field mode and its mode volume $V_{e f f}{ }^{28,29}$. The Q factor of a mode is calculated as $Q=2 \pi \frac{W}{\Delta W}$ where $W$ is the total (excess) average energy stored in the nanostructure and the electric field modified due to the presence of the nanostructure and $\Delta W$ is the average energy loss by the nanostructure per optical cycle. The excess energy is the energy difference between the space with and without the nanoparticle where we used boundary conditions of a plane wave in order to calculate the electric field. The energy stored in the nanostructure is the sum of the electric field energy in the space surrounding the particle and the energy stored in each of the constituent materials $W_{1,2}$ given by:

$$
W_{1,2}=\int_{V_{1,2}} w_{1,2}(r) d V
$$

where the energy density $w_{1, \mathbf{2}}(r)$ in each material $(1,2)$ is determined by its complex dielectric function $\varepsilon_{1, \mathbf{z}}$ and the electric field $\boldsymbol{E}_{\mathbf{1}}(\mathbf{1}, \mathbf{2})(\boldsymbol{r})$ in that material ${ }^{30}$.

$$
w_{1,2},(r)=\frac{1}{4} R e\left(\frac{d\left(\omega \varepsilon_{1,2}\right)}{d \omega}\right)\left|E_{1,2}(r)\right|^{2}
$$

The power loss is the sum of the Ohmic lossess in both materials and radiative losses . The Ohmic loss in each material is given by $P_{1,2}=\int_{V_{1,2}} p_{1,2}(r) d V$, where $p_{1,2}(r)=\frac{1}{2} \sigma_{1,2}\left|E_{1,2}(r)\right|^{2}$ and $\sigma_{1,2}=\omega \operatorname{lm}\left(\varepsilon_{1,2}\right)$. The radiative loss is given by $P_{\text {rad }}=\frac{\omega k^{\mathbf{3}} m^{2}}{12 \pi \varepsilon_{0}}$, where $m$ is the induced dipole moment in the nanostructure, ${ }^{k}$ is the wavevector of light in free space and $\varepsilon_{0}$ is the permeability of vacuum.

The mode volume $V_{\text {eff }}$ is given by

$$
V_{\text {eff }}=\frac{\int w_{\text {E }}(\boldsymbol{r}) d V}{\max (w(\boldsymbol{r}))}
$$

Here $w(r)$ denotes the energy density $w_{1,2}(r)$ in each material, as appropriate, and the integration is carried out over the whole space.

The FEM simulations of the $\mathrm{Q}$ factor and mode volume for the examined structures is shown in Figure 2. These are calculated at the fluorophore wavelength of $615 \mathrm{~nm}$, and using the refractive index of silica of $1.47^{11}$. The values of Q factor in the visible frequency range were surprisingly high, up to 250, due to low losses in silver. The mode volumes were very small, in the range of $10^{-5}-10^{-4} \mathrm{~nm}^{3}$. The value of Purcell factor $F$ predicted by these simulations varied between 1670 and 16600, suggesting large spontaneous emission enhancements for optical emitters placed inside the cavities formed by the nanoparticles under investigation. These values of the Purcell factor were comparable with other 
similar results in the literature. For example, for a plasmonic nanodisk resonator $\sim 1$ um in diameter, with two $100 \mathrm{~nm}$ Ag plates separated by $\sim 50 \mathrm{~nm}$ dielectric, the Purcell enhancement factor of over 2000 has been found ${ }^{25}$.

In order to compare these results with experiment we need to carry out appropriate averaging and corrections to account for location and other properties of the fluorophore ${ }^{29}$. The observed radiative lifetime, ${ }^{\tau}$, is reduced compared to the free space lifetime $\tau_{\text {free }}$, in the following way:

$$
\frac{\tau_{f r e e}}{\tau}=\frac{3 Q\left(\frac{\lambda_{c}}{n}\right)^{3}}{4 \pi^{2} V_{\text {eff }}} \frac{|E(r)|^{2}}{\left|E_{\max }\right|^{2}} \eta^{2}
$$

Here the factor $\eta=\boldsymbol{d} * \frac{\boldsymbol{E}(\boldsymbol{r})}{|\boldsymbol{d}|} * \mid \boldsymbol{E}(\boldsymbol{r}) \mathbf{|}$ describes orientational matching of the dipole moment and the electric field at the fluorophore location $\boldsymbol{r}$. The dipole associated with the fundamental optical transition of the fluorophore is randomly oriented so that the averaged $\eta^{2}=1 / 3$. Additionally, the spontaneous emission mode is twofold degenerate, producing a factor of $2^{31}$. We also need to average over all the positions of the fluorophores. Thus the free radiative $\tau_{\text {free }}$ and that in the presence of cavity, ${ }^{\tau}$, are related by:

$$
\frac{\tau_{f \text { ree }}}{\tau}=\frac{2^{3}}{3} \frac{\left(\frac{\lambda_{c}}{n}\right)^{3}}{4 \pi^{2} V_{e f f}}\left\langle\frac{|E(r)|^{2}}{\left|E_{\max }\right|^{2}}\right\rangle=\frac{2}{3} F\left(\frac{|E(r)|^{2}}{\left|E_{\max }\right|^{2}}\right\rangle
$$

where the term $\left(\frac{|E(r)|^{2}}{\left|E_{\max }\right|^{2}}\right\rangle$ expresses the average electric field experienced by the fluorophores located inside the second shell relative to the maximum field. We note here that some authors include the term $\frac{\tau_{\text {free }}}{\tau_{\text {leak }}}$ that accounts for the fact that the fluorophore in a cavity also radiates out into free space ${ }^{29}$. This is ignored here, because for low $Q$ cavities, the distinction between strictly cavity modes and strictly free-space modes is less distinct than in high Q dielectric cavities, and all modes are, to some extent, modified by the cavity. The fluorophore is assumed to interact with a single mode only, at the fluorophore emission frequency, this is appropriate for narrow spectral emission discussed here, but can be easily generalised.

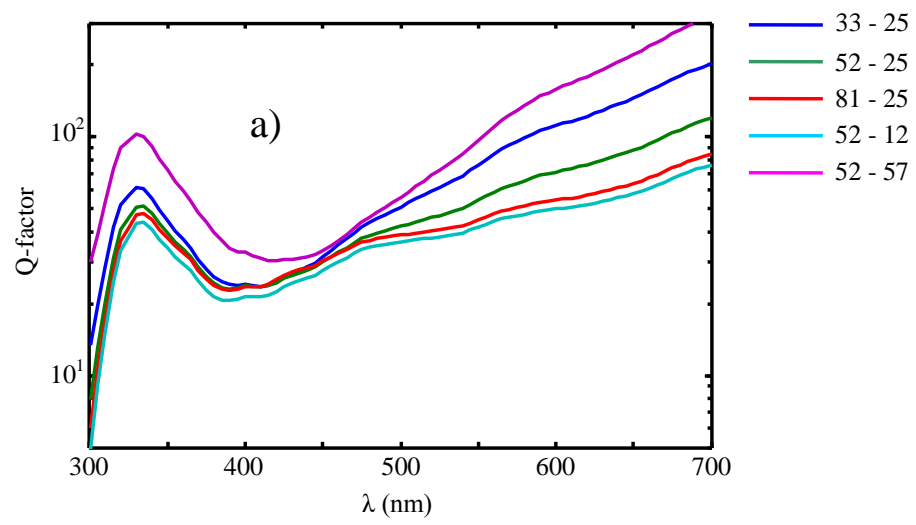




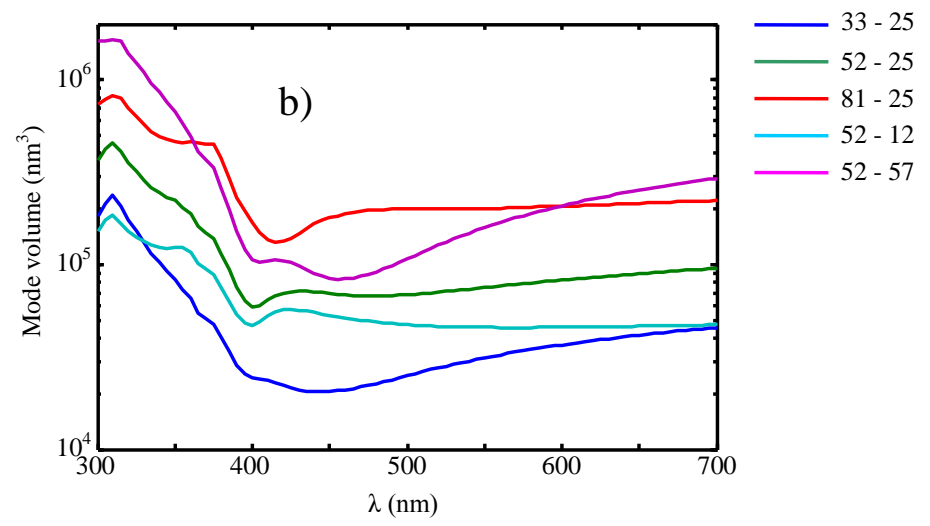

Figure 2. a) Calculated Q factors of the nanostructures at visible wavelength range, geometries indicated by different colors, as on the right, b) calculated mode volumes for the same samples. shell of $25 \mathrm{~nm}$, different core radii.

The above formalism is concerned with properties of electromagnetic modes in the cavity and its influence on radiative decay and this approach ignores the specific circumstances of a fluorophore. In order to account for the possibility of direct absorption of fluorophore emission by metal and nonradiative decay, we introduce a phenomenological quality factor of a fluorophore $Q_{\text {fivo }}{ }^{28}$. These properties of the fluorophore modifiy the quality factor by ${ }^{28}$ :

$$
\frac{1}{Q_{\text {eff }}}=\frac{1}{Q}+\frac{1}{Q_{\text {fluo }}}
$$

This effective quality factor is then used to calculate the effective Purcell factor

$$
F_{\text {eff }}=\frac{3 Q_{\text {eff }}\left(\frac{\lambda_{c}}{n}\right)^{3}}{4 \pi^{2} V_{e f f}}
$$

The fluorescence lifetime data together with the electric field in the structures calculated by using the Mie theory were used to compute the experimentally observed Purcell factors $F_{\text {exp } \bar{E}}$ observed in the nanostructures (Table 1). For the lifetime ratio $\frac{\tau_{\text {free }}}{\tau}$ we used the ratio of lifetimes observed in our composites to the lifetime observed in identical nanoshells (212 us) with the Lorentz local field correction given by $x=\frac{n\left(n^{2}+2\right)^{2}}{9}$, where $n$ is the value of refractive index in silica. Such approach addresses the influence of the solvent quenching which should similarly affect the composite nanoparticles and nanoshells. Our values of ${ }^{F_{\exp }}$ have been obtained by using electric field given by the exact solution of the Maxwell's equations within Mie theory, The results shown in Table 1 indicate that both our lifetime ratios and experimental estimates of the Purcell factor $F_{\text {exp }} \bar{E}$ are high, and comparable to those observed in good quality micropillar cavities with high $Q$ examined by Gerard et al. ${ }^{7}$. This is despite much lower $Q$ factor in our nanostructures, which is compensated by very low mode volumes. The discrepancy between the values of $F_{\exp } \bar{E}$ and ${ }^{F}$ is related to the dissipation of energy by a fluorophore by processes other than cavity resonance (such as nonradiative decay). These are strongest in smallest nanoparticles and those where fluorophores are closest to the metal, consistent with accepted notions about energy dissipation in such plasmonic systems. 


\section{$|E(r)|^{2}$}

Table 1. Observed lifetimes $\tau$, average electric fields at fluorophore locations $\overline{\left|E_{\max }\right|^{2}}$, theoretical $Q$ factor and mode volume, $V_{\text {eff }}$ at $615 \mathrm{~nm}$, effective fluorophore quality factor $Q_{\text {flwo }}$ providing match of Equation 10 with experimental values of Purcell factor $F_{\exp } \bar{E}$. Also theoretical Purcell factor ${ }^{F}$ using Equation 2. The data for 52/25 nanoparticle have been repeated for clarity.

\begin{tabular}{|c|c|c|c|c|c|c|c|c|c|}
\hline $\begin{array}{c}\text { Ag core diameter } \\
/ \mathrm{SiO}_{2} \text { shell thickness } \\
(\mathrm{nm})\end{array}$ & $\begin{array}{ll}\tau & \text { [us] }\end{array}$ & $\begin{array}{c}\frac{\tau_{\text {free }}}{\tau} \\
\tau_{\text {free }} \stackrel{\tau}{=} \\
\text { us }\end{array}$ & $\frac{|E(r)|^{2}}{\left|E_{\max }\right|^{2}}$ & $F_{\text {exp } \bar{E}}$ & $Q$ & $\begin{array}{l}V_{\text {eff }} \\
\left(\mathrm{nm}^{3}\right)\end{array}$ & $F$ & $Q_{\text {eff }}$ & $Q_{\text {fluo }}$ \\
\hline $81 / 25$ & $119+/-2$ & 5.3 & 0.11 & 72 & 60 & $2 \times 10^{5}$ & 1669 & 2.58 & 2.69 \\
\hline $52 / 25$ & $101+/-2$ & 6.3 & 0.11 & 86 & 80 & $9 \times 10^{4}$ & 4945 & 1.39 & 1.41 \\
\hline $33 / 25$ & $185+/-3$ & 3.4 & 0.11 & 46 & 120 & $4 \times 10^{4}$ & 16600 & 0.33 & 0.33 \\
\hline $52 / 12$ & $60+/-3$ & 10.6 & 0.16 & 99 & 52 & $5 \times 10^{4}$ & 5786 & 0.88 & 0.89 \\
\hline $52 / 25$ & $101+/-2$ & 6.3 & 0.11 & 86 & 80 & $9 \times 10^{4}$ & 4945 & 1.39 & 1.41 \\
\hline $52 / 57$ & $161+/-3$ & 3.9 & 0.084 & 70 & 170 & $2 \times 10^{5}$ & 4729 & 2.52 & 2.55 \\
\hline
\end{tabular}

\subsection{Criteria for lasing}

The presence of a resonant cavity with a comparatively high quality factor compared with metal nanoparticles and high Purcell factors indicate the possibility of lasing in such systems. The spontaneous emission coupling efficiency factor $\beta=F / F+1$ in such structures is close to unity and this has implications for low threshold lasing. In order to understand the lasing process we draw analogies with conventional laser cavities. The plasmonic nanoparticle localizes the optical wave around the metal nanostructure and induces phase shifts, thus acting like an optical cavity. The onset of lasing is possible only at those frequencies of electromagnetic field where the structure experiences a resonance condition (eigenmode). We note that a core-shell plasmonic nanoparticle with a metal core exhibits a number of eigenmodes (dipole and higher multipoles) of electromagnetic field, although some of them, especially the multipole modes are characterized by large width. Furthermore, laser action is possible only at frequencies where the medium (small-signal) gain exceeds the loss coefficient. This condition selects the cavity mode where lasing is initiated with increasing gain. These ideas are now used to establish the conditions for lasing in composite plasmonic nanoparticles.

The formalism used here is based on exact analytical solutions of the Maxwell equations in spherical geometry and it combines the Mie theory and T-matrix method ${ }^{32,33}$. The calculations shown here were carried out for core-shell structures with the arrangement of layers shown in Figure 3a and they can be easily extended to structures with an arbitrary number of concentric spherical shells. In our calculations the inner sphere was made of a noble metal Au or Ag and the outer shell was a dielectric able to provide gain that is uniformly distributed across the structure. The dielectric function in the metal (gold or silver) was taken from Ref. 14. The dielectric function in the dielectric, $\varepsilon_{\mathrm{r}}-i \varepsilon_{\mathfrak{i}}$, was assumed to have an additional negative imaginary term responsible for gain.

In order to calculate the threshold gain for lasing one needs to consider the characteristics of the eigenmodes of the structure in the presence of gain. To this aim we evaluated the resonance frequencies of the dipolar and higher modes by finding nontrivial solution of the Maxwell's equations that is finite at the centre of the core and far from the centre contains only the outgoing wave that tends to zero at infinity. The lasing frequency is determined by solving a system of equations derived from matching the tangential components of the electric and magnetic fields at the boundaries of the inner and outer surface of the spherical shell, in a way similar as in References 34 and 35. This matching is possible only for selected values of the wave vector of the spherical wave, which specifies the threshold lasing condition for the real and imaginary part of a dielectric function, at each frequency.

The value of the refractive index of a dielectric at zero gain determines the eigenmode frequency where lasing is possible for a given structure geometry. This is illustrated in Figure $3 \mathrm{~b}$ where we show the refractive index of the dielectric at zero gain, $\sqrt{\varepsilon_{r}}$, for different eigenmodes and the corresponding values of imaginary part of the refractive index 
$k=2^{-\frac{1}{2}} \sqrt{-\varepsilon_{r}+\sqrt{\varepsilon_{i}^{2}+\varepsilon_{r}^{2}}}$

as a function of lasing wavelength. The latter is related to the optical gain coefficient by $g=\mathbf{4} \pi k / \lambda$ Our calculations shown in Figure $3 \mathrm{~b}$ have been carried out for the nanoparticle geometry identical to that reported by Noginov ${ }^{36}$. The lasing is taking place in a dipolar mode and we note that our predictions of the lasing wavelength for this geometry $(527.3 \mathrm{~nm})$ corresponds closely to $531 \mathrm{~nm}$ measured in Noginov's experiment.

Supported with this experimental validation of our model we have extended our simulations to other similar geometries within the experimentally achievable range of sizes. In these simulations we used both gold and silver while the refractive index of the gain medium was taken as that of silica ${ }^{11}$. The geometries discussed here are listed in Table 2.

Table 2. Details of the examined nanostructure geometries, all with $\mathrm{SiO}_{2}$ shells and their lasing characteristics.

\begin{tabular}{|l|l|l|l|l|l|l|}
\hline $\begin{array}{l}\text { Core } \\
\text { material }\end{array}$ & $\begin{array}{l}\text { Core } \\
\text { diameter, } \\
d=2 R_{1} \\
(\mathrm{~nm}),\end{array}$ & $\begin{array}{l}\text { Shell } \\
\text { thickness } \\
t=R_{2}-R_{1} \\
(\mathrm{~nm})\end{array}$ & Lasing mode & $\begin{array}{l}\text { Lasing } \\
\text { waveleng } \\
\text { th } \\
(\mathrm{nm})\end{array}$ & $\begin{array}{l}\text { Imaginary part of the } \\
\text { refractive index } k \text { at } \\
\text { threshold }\end{array}$ & $\begin{array}{l}\text { Threshold gain coefficient } \\
g=\mathbf{4 \pi k} / \lambda \\
\left(\mathrm{cm}^{-1}\right)\end{array}$ \\
\hline $\mathrm{Au}$ & 14 & 15 & Dipolar & 527.3 & 0.41 & $9.77 \times 10^{4}$ \\
\hline $\mathrm{Au}$ & 50 & 50 & Dipolar & 542.8 & 0.30 & $6.97 \times 10^{4}$ \\
\hline $\mathrm{Ag}$ & 14 & 15 & Dipolar & 399.9 & 0.038 & $1.19 \times 10^{4}$ \\
\hline $\mathrm{Ag}$ & 30 & 15 & Quadrupolar & 378.7 & 0.043 & $1.43 \times 10^{4}$ \\
\hline $\mathrm{Ag}$ & 50 & 50 & Quadrupolar & 383.3 & 0.038 & $1.25 \times 10^{4}$ \\
\hline
\end{tabular}

The first geometry with a $14 \mathrm{~nm}$ diameter core $(d=14 \mathrm{~nm})$ and a silica shell with thickness $t=15 \mathrm{~nm}$ is identical to that shown to lase in Ref. 36. The value of threshold gain for this structure is predicted to be $9.77 \times 10^{4} \mathrm{~cm}^{-1}$. The conditions for lasing are somewhat more favorable in structures with a large core diameter and a thicker shell $(d=50 \mathrm{~nm}$ and $t=50$ $\mathrm{nm}$, respectively), where the threshold gain of $6.97 \times 10^{4} \mathrm{~cm}^{-1}$ is found. Here the lasing mode spreads out over a thicker shell and its smaller fraction extends over the lossy core region compared to the previous geometry $(d=14 \mathrm{~nm}, t=15 \mathrm{~nm})$. For this large structure the lasing wavelength is also comparatively longer (542.8 nm). This longer lasing wavelength is further away from the energy-dissipating interband transitions in the metal, hence favorable influence on the laser threshold. 
a

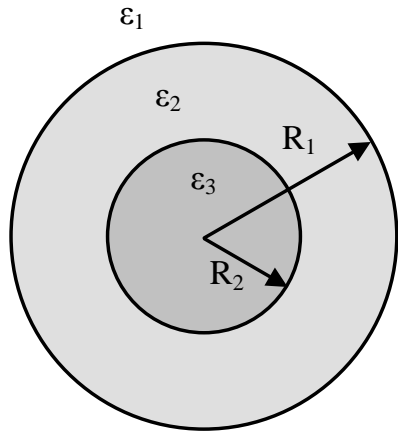

C

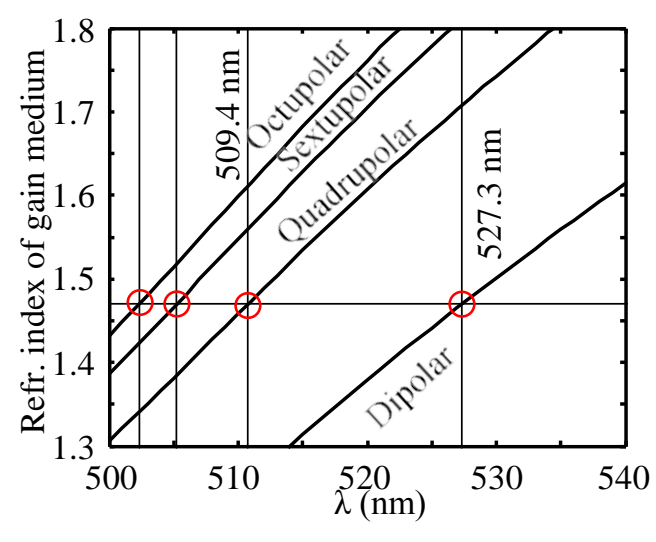

e

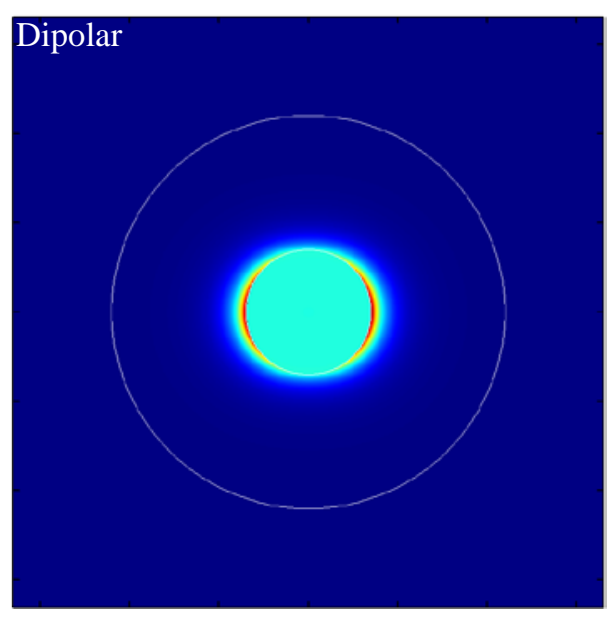

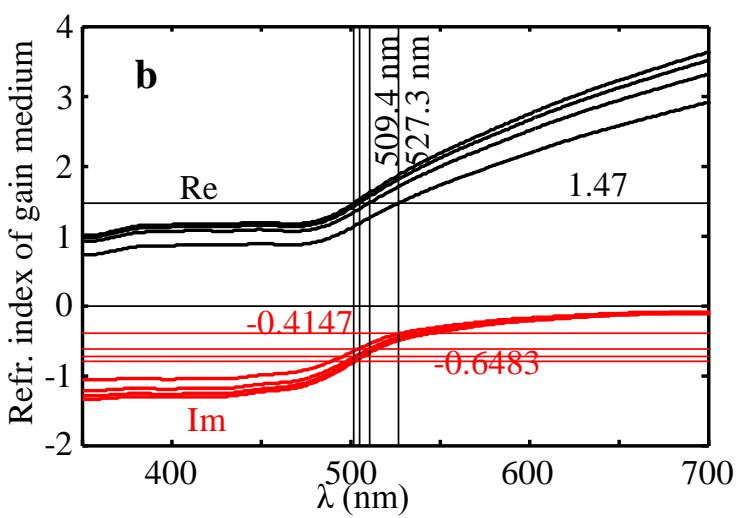

d

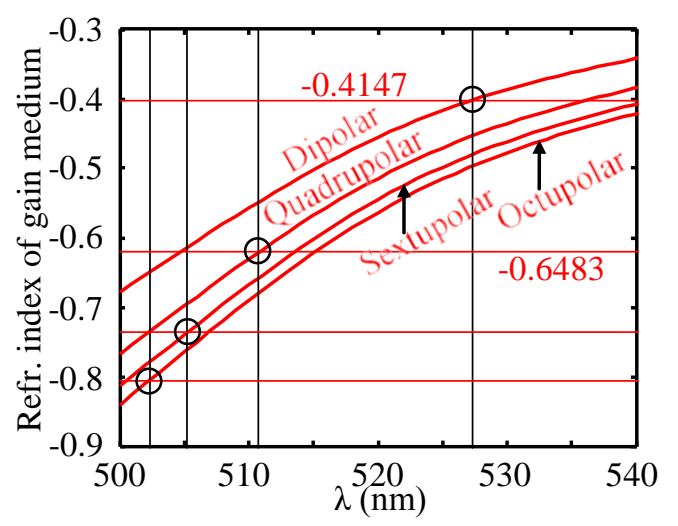

f

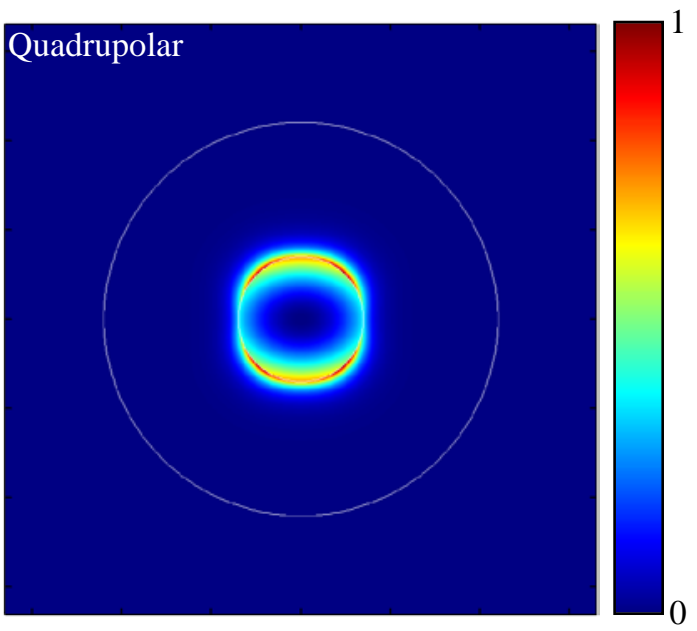

Figure 3. a) The core-shell geometry of the nanostructures. b-e) results obtained for the $\mathrm{d}=14, \mathrm{t}=15 \mathrm{~nm}$ Au core structure as in Ref. 1 , b) The real part of the refractive index at zero gain, $\sqrt{\varepsilon_{\mathbf{r}}}$, and the imaginary part of the refractive index in the presence of gain, $\mathrm{k}$, as a function of wavelength for the lowest few eigenmodes. The horizontal line at the refractive index of silica ( $\mathrm{n}=1.47$ ) identifies the mode frequency. The mode with the smallest $|\mathrm{k}|$ is the first lasing mode. $\mathrm{c}$ ) and d) magnified view of the panel b) showing the frequencies of the eigenmodes and the corresponding values of $k$ for different modes. e) and $f$ ) the calculated patterns of local field intensity in the lowest modes, a)dipolar, b) quadrupolar. 
The inspection of the nanoparticles with Ag core leads to somewhat different observations. The structure with $d=14 \mathrm{~nm}$, $t=15 \mathrm{~nm}$ is lasing in a dipolar mode, at a much shorter wavelength of $399.9 \mathrm{~nm}$ than the corresponding Au core structure, with a very small threshold gain of $1.19 \times 10^{4} \mathrm{~cm}^{-1}$. This is due to smaller losses in Ag than in Au. However even a small increase of the core diameter, to $30 \mathrm{~nm}$, changes the lasing mode from dipole into the quadrupole, with further decrease of the lasing wavelength to $378.7 \mathrm{~nm}$, and increased threshold gain to $1.43 \times 10^{4} \mathrm{~cm}^{-1}$. This mode switch is induced by the fact that in the quadrupole mode the electric field is concentrated rather in the outer part of the metal core, which lowers the losses. Upon further increase of core and shell size, to $d=50 \mathrm{~nm}, t=50 \mathrm{~nm}$ the lasing continues in the quadrupole mode and the lasing wavelength increases to $383.3 \mathrm{~nm}$ while the threshold gain decreases somewhat, to $1.25 \times 10^{4} \mathrm{~cm}^{-1}$ (data not shown).

Our results show that all the examined nanoparticles are capable of lasing, provided the threshold gain $g$ can be achieved by using suitable fluorophores. Silver core appears especially favourable for lasing as, due to smaller losses in Ag than $\mathrm{Au}$, the threshold gain for silver is about one order of magnitude lower than in gold with values between $1.19 \times 10^{4} \mathrm{~cm}^{-1}$ and $1.43 \times 10^{4} \mathrm{~cm}^{-1}$ (Table 2). The gain coefficient is a product of concentration, $N$ and the emission cross section, $\sigma_{e m}$ of gain molecules, $g=N \sigma_{e m}$. With the current progress in chemistry of covalent-binding of fluorophores into silica nanoparticles chemistry the concentrations in the order of $\sim 6.25 \times 10^{19} \mathrm{~cm}^{-3}$ have been achieved. To reach the lasing threshold the UV emitting dyes are required with an emission cross-section of $2.28 \times 10^{-16} \mathrm{~cm}^{2}$ at $399.9 \mathrm{~nm}$ or $1.91 \times 10^{-16} \mathrm{~cm}^{2}$ at $378.8 \mathrm{~nm}$. Although the number of fluorescent dyes emitting between $370 \mathrm{~nm}$ to $400 \mathrm{~nm}$ is limited and they are relatively weak comparing with the visible dyes, their emission cross-sections are sufficient. For example, Methoxycoumarin dyes (succinimidyl ester with excitation peak at $\sim 350 \mathrm{~nm}$; emission peak $380 \mathrm{~nm}$; Invitrogen), capable of binding to APS and compatible with the current covalent-binding silica nanoparticle chemistry have absorption cross section of in excess of $1.6 \times 10^{-16} \mathrm{~cm}^{236,37}$.

\section{4.}

\section{CONCLUSIONS}

This work is concerned with Ag-core/silica-shell nanocomposites with an Eu-chelate BHHCT-Eu-DPBT introduced into a well defined external region of the silica shells of $\mathrm{Ag} / \mathrm{SiO}_{2}$ core-shell nanostructures. These plasmonic nanoparticles of spherical geometry (50-100 nm in diameter) are too small to accommodate whispering gallery modes, but they support dipolar, quadrupolar and higher modes of electromagnetic field. They also concentrate and enhance field in their proximity, modifying the local density of states, and inducing the Purcell effect. We have observed this effect through enhancement of fluorescence and lifetime reduction of Eu fluorophores introduced into the outer layer of the silica shell. The Fermi Golden Rule, rewritten in the Purcell formalism has been used to interpret the results. The Purcell formalism is particularly convenient as it makes it possible to separate out the effects that relate to the nanostructure geometry from those that are related to the fluorophore. Numerical simulations of the Q factor and mode volume of the plasmonic core-shell nanocomposite particle, within the Mie theory and by the finite element revealed a very high Purcell factor of the cavities alone $(>4000)$ and a smaller effective Purcell factor when the fluorophore properties such as nonradiative recombination are taken into consideration.

Finally, we described how to calculate the lasing frequency of composite core-shell noble metal-dielectric nanoparticles. We examined several core shell geometries with varying sizes and established trends for the lasing frequency and threshold gain.

Optical cavities interacting with two-level systems are intensely studied in classical, quantum optics and nanooptics, especially in the high Purcell factor regime. Our work demonstrates that metal/dielectric core shell structures, with their moderate Q and ultrasmall mode volumes offer a technically simple and appealing alternative to high Q micropillar and photonic crystal cavities. Such structures can serve as a testing ground for fundamental studies of interactions between light and matter, and they show enhanced fluorescence and modified fluorescence lifetimes, important for applications. We can then expect interesting possibilities for the application of these and similar composites as novel light sources. 


\section{REFERENCES}

[1] W. E. Moerner and M. Orrit, Science 283, 1670 (1999).

[2] D. C. Unitt et al., J. Opt. B 7, S129 (2005).

[3] S. Lal et al., Nat. Photon. 1, 641 (2007).

[4] G.A. Wurtz et al, Nano letters, 7, 5, 1297-1303, (2007)

[5] E. Feigenbaum and M. Orenstein, Phys. Rev. Lett., 101, 163902 (1-4) (2008).

[6] S.A. Meier, Optical and Quantum Electron., 38, 257-267 (2006), S.A. Meier, Optics Express, 14,5, 1957-1964, (2006)

[7] J.M. Gerard, et al., Phys Rev. Lett. 81,5, 1110-1113, (1998)

[8] J. Wu et al. J. Mater. Chem. 19, 1258 - 1264, (2009)

[9] P.C. Lee, D. Meisel,. J. Phys. Chem., 86, 3391-3395, (1982)

[10] S. Liu, Z. Zhang, M. Han, Anal. Chem., 77, 2595-2600, (2005)

[11] Handbook of Optical Constants of Solids edited by E. D. Palik, Academic Press, New York, 1985, for silica see also Smithsonian Physical Tables (9th Revised Edition): Forsythe, W.E., 518, (2003).,26, 27

[12] A.L. Aden, M. Kerker, J. Appl. Phys. 22, 10, 1242-1246, (1951).

[13] J.A. Gordon, R.W. Ziolkowski, Optics Express, 15, 5, 2622-2653, (2007).

[14] Johnson P.B., Christy R.W., Phys. Rev. B 6, 4370, (1972).

[15] F. Hao, P. Nordlander, “, Chemical Physics Letters, 446, 115-118, (2007).

[16] F.J. Garcia de Abajo, J. Phys. Chem. C, 112, 17983-17989, (2008)

[17] T.V. Teperik, V.V. Popov, F.J. Garcia de Abajo, Phys. Rev. B 69, 155402 (2004).

[18] J.R. Lakowicz, Analytical Biochemistry, 1, 298, 1-24 (2001)

[19] W. Deng et.al, , Langmuir 2010, 26, 1048-1077.

[20] K. Sokolov, G. Chumanov, T Cotton, Anal. Chem 1998, 70, 3898-3905.

[21] K. Aslan et.al. , Curr Opin Biotechnol 2005, 16, 55-62.

[22] E. M. Purcell, Phys. Rev. 69, 37 (1946).

[23] J.M. Gerard et al. Appl. Phys. Lett, 69,4,449-451 (1996)

[24], M. Barth et al, Nano Letters, 10, 891-895 (2010)

[25] M. Kuttge, F.J. Garcia de Abajo, A. Polman, Nano letters, 10, 1537-1541 (2010)

[26] H. Iwase, D. Englund, J. Vuckovic, Optics Express, 18,16, 16546-16560, (2010), also V.J. Sorger, R.F. Oulton, J. Yao, G. Bartal, Nano letters, 9,10, 3489-3493 (2009)

[27] E. Feigenbaum, M. Orenstein, Phys. Rev. let, 101, 163902-1-4, (2008)

[28] M. Boroditsky et al. J. Lightwave Technol. 17,11, 2096 (1999)

[29] J.M. Gerard, B. Gayral, Journal of Lightwave Technology, 17, 11, 2089-2095, (1999)

[30] R. Ruppin, Physics Letters A 299, 309-312, (2002). For discussion of mode volume, see A.F. Koenderick. Opt. Lett. 35,24, 4208-4210, (2010).

[31] J.M. Gerard, B. Gayral, Phys. Rev. Lett. , 90, 22, 229701-1, (2003)

[32] Stratton J.A., “Electromagnetic Theory”, McGraw Hill NY, 563-573, (1941). 
[33] Teperik T.V., Popov V.V., Garcia de Abajo F.J., Phys. Rev. B 69, 155402 (2004).

[34] Aden A.L., Kerker M., J. Appl. Phys. 22, 10, 1242-1246, (1951).

[35] J.A. Gordon, R.W. Ziolkowski , Optics Express, 15, 5, 2622-2653, (2007).

[36] M.A. Noginov., et al. ,Nature , 460, 7259, 1110-1112 (2009).

[37]L. Wang, L. et al. , Analytical Chemistry, 78, 646-654 (2006).

[38] J. Wu, et al., Chemical Communications, 365-367 (2008). 